\title{
Impact of early detection and treatment of diabetes on the 6-year prevalence of cardiac autonomic neuropathy in people with screen-detected diabetes: ADDITION-Denmark, a cluster-randomised study
}

\author{
M. Charles $\cdot$ J. Fleischer $\cdot$ D. R. Witte $\cdot$ N. Ejskjaer • \\ K. Borch-Johnsen • T. Lauritzen • A. Sandbaek
}

Received: 18 June 2012 / Accepted: 12 September 2012 /Published online: 12 October 2012

(C) Springer-Verlag Berlin Heidelberg 2012

\begin{abstract}
Aims/hypothesis There is limited evidence on how multifactorial treatment improves outcomes of diabetes when initiated in the lead time between detection by screening and diagnosis in routine clinical practice. Cardiac autonomic neuropathy $(\mathrm{CAN})$ in people with diabetes indicates widespread damage to the autonomic nervous system, which may severely affect health and quality of life. We examined effects of early detection and subsequent intensive treatment of type 2 diabetes in primary care on the prevalence of CAN at the 6-year follow-up examination in a pragmatic clusterrandomised parallel group trial.

Methods One hundred and ninety general practices were randomised to deliver either intensive multifactorial treatment (IT)
\end{abstract}

Electronic supplementary material The online version of this article (doi:10.1007/s00125-012-2744-5) contains peer-reviewed but unedited supplementary material, which is available to authorised users.

M. Charles $(\bowtie) \cdot$ T. Lauritzen $\cdot$ A. Sandbaek

Department of Public Health, Section of General Practice,

Bartholins Allé 2, bygn. 1260,

8000 Aarhus C, Denmark

e-mail: mc@alm.au.dk

J. Fleischer $\cdot$ N. Ejskjaer

Department of Endocrinology, Aarhus University Hospital,

Aarhus, Denmark

D. R. Witte

Steno Diabetes Center,

Gentofte, Denmark

K. Borch-Johnsen

Research Center for Quality in Health Care,

Institute of Public Health, University of Southern Denmark,

Odense, Denmark or routine care $(\mathrm{RC})$ as recommended by national guidelines to patients with type 2 diabetes, identified through a stepwise screening programme in the primary care setting. 1533 people (IT, $n=910 ; \mathrm{RC}, n=623$ ) were identified and included. At the 6-year follow-up examination, measures of CAN were applied in an unselected subsample of 777 participants using heart rate variability analysis and standard tests of CAN.

Results At the 6-year follow-up examination, the prevalence of early CAN was $15.1 \%$ in the RC group and $15.5 \%$ in the IT group, while manifest CAN was present in $7.1 \%$ and $7.3 \%$, respectively. We found no statistically significant effect of intensive treatment on the prevalence of CAN compared with routine care.

Conclusions/interpretation In the Danish arm of the ADDITION Study, signs of CAN were highly prevalent 6 years after a screening-based diagnosis of type 2 diabetes. Intensive multifactorial treatment did not significantly affect the prevalence of CAN compared with routine care. However, at followup the level of medication was also high in the RC group.

Keywords ADDITION · Cardiac autonomic neuropathy · Diabetic neuropathy $\cdot$ Heart rate variability $\cdot$ Prevalence . Screen-detected diabetes $\cdot$ Trial

$\begin{array}{ll}\text { Abbreviations } \\ \text { ADDITION } & \begin{array}{l}\text { Anglo-Danish-Dutch Study of Intensive } \\ \text { Treatment in People with Screen-Detected } \\ \end{array} \\ & \begin{array}{l}\text { Diabetes in Primary Care } \\ \text { Cardiac autonomic neuropathy }\end{array} \\ \text { CAN } & \text { Diabetic peripheral neuropathy } \\ \text { DPN } & \text { Heart rate variability } \\ \text { HRV } & \text { Intensive multifactorial treatment } \\ \text { IT } & \text { Routine care }\end{array}$




\section{Introduction}

Screening for diabetes is advocated in many countries. However, there is limited evidence that benefit estimates obtained in studies of clinically detected type 2 diabetes also apply to screen-detected populations. Cardiac autonomic neuropathy (CAN) in patients with diabetes is associated with an increased mortality risk compared with patients with diabetes and no signs of CAN, but it remains an overlooked complication of diabetes, and the prevalence among people with screen-detected diabetes is unknown [1]. Furthermore, there is limited trial evidence on prevention of CAN. The Steno-2 Trial showed that, in patients with longstanding type 2 diabetes, intensive multifactorial intervention can reduce the risk of developing CAN by $68 \%$, while there was no reduction in the risk of diabetic peripheral neuropathy (DPN) [2].

The multicentre Anglo-Danish-Dutch Study of Intensive Treatment In People with Screen-Detected Diabetes in Primary Care (ADDITION) Trial was the first trial to study the effect of early detection of type 2 diabetes in general practice followed by intensive multifactorial treatment based on the treatment principles of the Steno-2 Trial [3]. In a previous analysis, we found that intensive multifactorial treatment led to a statistically non-significant reduction in the prevalence of DPN compared with routine care as recommended by national guidelines [4].

In this paper we aim to describe the effect of early detection and consecutive routine care or intensive multifactorial treatment on the prevalence of CAN in patients with screen-detected type 2 diabetes at the 6-year followup examination.

\section{Methods}

Design The design and rationale of the ADDITION Study (ClinicalTrials.gov NCT00237549) have been reported [5]. In brief, ADDITION-Denmark consists of two phases: (1) a screening phase; and (2) a pragmatic, cluster-randomised parallel group trial. In five regions of Denmark, 190 general practices were randomised to screening plus routine care of diabetes (RC) or screening followed by intensive multifactorial treatment (IT). Randomisation was stratified by region and the number of full-time general practitioners per practice.

A population-based stepwise screening programme among people aged 40-69 years without known diabetes was undertaken in each practice, and individuals were diagnosed with diabetes according to WHO criteria, as previously described [6]. Overall, 1533 (623 RC, 910 IT) eligible participants with screen-detected diabetes agreed to take part in the trial. After an average of 5.9 years of follow-up, 1278 participants were re-examined (512 RC, 766 IT). Of these, 108 were only seen by their own general practitioner. Examinations for CAN were performed in three of the test centres (863 participants; 339 RC, 524 IT). Sixty-four participants attending one of these centres were not tested for CAN. Reasons for missing measurements were: station closure because of insufficient staff or unavailable testing device $(n=42)$; atrial fibrillation or pacemaker $(n=14)$; disability preventing testing $(n=3)$. Of a total of 799 participants tested for CAN, 22 had missing values on at least two of the three standard CAN tests mentioned below, and 76 had a missing value in one of three. Most missing values were due to difficulties in complying with the strain pressure in the Valsalva manoeuvre or with rising from the supine position to standing. As two abnormal tests are sufficient for a diagnosis of CAN [7], we chose to include all participants with at least two complete tests, yielding a study sample of 777 participants in this analysis. Electronic supplementary material (ESM) Fig. 1 displays the practice and participant flow.

Intervention The specific characteristics of the interventions to promote intensive treatment have been described previously in detail [5]. The purpose of the intensive treatment was to provide the best possible evidence-based treatment in primary care. We aimed to educate and support general practitioners and practice nurses in target-driven management (using medication and promotion of a healthy lifestyle) of hyperglycaemia, blood pressure and cholesterol, based on the stepwise regimen used in the Steno-2 Study [2]. Intensive treatment was promoted through the addition of several features to existing diabetes care. Practice staff were provided with educational materials for patients, and patients were sent reminders if annual check-up appointments were overdue. Practices received additional funding to support the delivery of the extra care added to the usual care/consultations. All treatment targets and algorithms were based on evidence from randomised controlled trials demonstrating the benefits of intensive treatment of cardiovascular risk factors in people with type 2 diabetes [3] and are displayed in ESM Table 1. Family physicians were advised to consider prescribing ACE inhibitors or angiotensin II antagonists for patients who had a blood pressure of $120 / 80 \mathrm{mmHg}$ or higher, and $75 \mathrm{mg}$ aspirin daily for patients who had no specific contraindications. After publication of the Heart Protection Study, we altered the treatment algorithm to recommend prescription of a statin for all patients with a cholesterol concentration of $3.5 \mathrm{mmol} / \mathrm{l}$ or higher at any time. Although treatment targets were prespecified and classes of medication recommended, decisions on prescriptions, including the choice of individual drugs, were made by practitioners and patients.

In the RC group, general practitioners were only provided with diagnostic test results and patients received the 
standard pattern of diabetes care according to Danish national recommendations $[8,9]$.

Measurements Health assessments at baseline and followup included biochemical, anthropometric and questionnaire measures, and were undertaken by centrally trained staff unaware of study group allocation following standard operating procedures. Blood pressure was calculated as the mean of three measurements performed after at least a $10 \mathrm{~min}$ rest, using Omron blood pressure recorders. Height and weight were measured in light indoor clothing, without shoes, using a fixed rigid stadiometer and a Tanita scale, respectively.

Biochemical measurements were performed at Aarhus University Hospital and Steno Diabetes Centre, Gentofte at baseline and follow-up, respectively. The specific analyses have been described previously [6].

At the 6-year follow-up examination, heart rate variability (HRV) on supine rest and standard tests of CAN were performed: after $10 \mathrm{~min}$ of supine resting, a 2 min ECG recording was made in all participants to obtain HRV. The beat-to-beat intervals in this $2 \mathrm{~min}$ ECG recording were analysed by normal statistical descriptions (time domain) and by estimating frequency-specific fluctuations in HRV (frequency domain) [10]. In the time domain, the root mean square of the sum of the squares of differences between consecutive $\mathrm{R}-\mathrm{R}$ intervals and standard deviation of normal-to-normal intervals were calculated. In the frequency domain, we calculated the power in the low-frequency $(0.04-0.15 \mathrm{~Hz})$ and high-frequency $(0.15-0.4 \mathrm{~Hz})$ band. These measures were included, as they can provide early additional information on both sympathetic and parasympathetic modulation [11].

Participants were subsequently tested with three standard CAN tests [12].

(1) Lying to standing (30/15). The participant was asked to quickly rise from the supine to the standing position as the ECG was continuously recorded, and the ratio between the shortest R-R interval (around the 15th heartbeat after the rise) and the longest $\mathrm{R}-\mathrm{R}$ interval (around the 30th heartbeat) was calculated [13]. A ratio with age-dependent cut-off levels was used: age $<30$ years, 1.15 ; age $30-39$ and $40-49$ years, 1.07; age 50-59 years, 1.05; age 60-69 years, 1.02; age 70-79 years, $1.00[12,14]$.

(2) Deep breathing for $1 \mathrm{~min}$ with a respiration frequency of six breaths/min to examine the exhalation/inhalation ratio (E:I ratio) [13]. The E:I ratio was expressed as the mean of the longest $\mathrm{R}-\mathrm{R}$ interval during each inhalation divided by the mean of the shortest $\mathrm{R}-\mathrm{R}$ interval during each exhalation. Age-dependent cut-off levels were used for the E:I ratio: age $<30$ years, 1.24; age $30-39$ years, 1.19 ; age $40-49$ years, 1.15 ; age 50
59 years, 1.11 ; age $60-69$ years, 1.08 ; age $70-79$ years, $1.05[12,14]$.

(3) Valsalva manoeuvre [13] to determine the ratio of maximum and minimum $\mathrm{R}-\mathrm{R}$ interval during forced expiration in $15 \mathrm{~s}$ against a fixed resistance and $45 \mathrm{~s}$ of normal breathing. Age-dependent cut-off levels were used: age $<30$ years, 1.42; age 30-39 years, 1.35; age 40-49 years, 1.29; age 50-59 years, 1.24; age 6069 years, 1.19; age $70-79$ years, $1.15[12,14]$.

Consumption of food and caffeine-containing beverages and smoking were prohibited for $2 \mathrm{~h}$ before laboratory testing. All laboratory tests for CAN were performed between 08:00 and 13:00 hours in a quiet and isolated examination room. HRV and the cardiovascular reflex tests were performed by technicians using a Vagus device (Medicus Engineering, Aarhus, Denmark) [15]. This device automatically records an ECG signal, with a sampling frequency of $1,000 \mathrm{~Hz}$, from which heart rate and HRV are calculated. CAN was defined using the ADA criteria and the Toronto Consensus Panel on Diabetic Neuropathy as follows: CAN 0 , all tests are normal; CAN early, one test is abnormal signifying the presence of possible or early stage CAN; CAN manifest, two or three abnormal tests signifying definite or late stage CAN $[12,16]$.

Standardised self-report questionnaires were used to collect information on sociodemographic characteristics (education, employment and ethnicity), lifestyle habits (smoking status, alcohol consumption) and self-reported cardiovascular disease (previous myocardial infarction, stroke or operation/instrumentation on the heart).

Statistical analysis Patient characteristics at baseline and follow-up are presented as unadjusted means, or in the case of skewed distributions as medians. Participants with missing values were compared with participants without missing values. The prevalence data for each treatment group of CAN were calculated as number of people with abnormal tests divided by number of people who participated in the test and had complete data from at least two CAN tests. Data are presented with $95 \%$ CIs. The median values of the CAN tests and the time and frequency measures were compared between treatment groups. HRV ordinal logistic regression was used to estimate the OR and $95 \%$ CI for the comparison of the IT group with the RC group. The outcome measure was presence of any degree of CAN. The standard errors were adjusted to allow for intracluster correlation, where the clusters were the general practices, using the 'cluster()' option in Stata. Interaction by sex, age, baseline $\mathrm{HbA}_{1 \mathrm{c}}$, total cholesterol and systolic blood pressure was examined with linear interaction terms between the treatment allocation and quartiles of each of these variables (except for sex). The effect of the intervention, expressed as an OR, is 
presented stratified by the treatment target recommended at trial initiation $\left(\mathrm{HbA}_{1 \mathrm{c}}<7 \%[53 \mathrm{mmol} / \mathrm{mol}]\right.$, total cholesterol $<5 \mathrm{mmol} / \mathrm{l}$, and systolic blood pressure $\leq 135 \mathrm{mmHg}$ ), sex and age (below/above 60 years). Sensitivity analyses were performed, excluding patients using $\beta$ blockers and patients who reported regular or frequent hypoglycaemia. Statistical analyses were performed with Stata (version 11, StataCorp LP, College Station, TX, USA).

\section{Results}

Baseline characteristics are shown in Table 1. The average follow-up time was 5.8 and 5.9 years for the RC and IT groups, respectively. Median $\mathrm{HbA}_{1 \mathrm{c}}$ did not change over the follow-up period in either group, but there was a similar decline in systolic blood pressure and total cholesterol in both groups. The number of smokers substantially declined in both groups with no difference between groups. Total alcohol consumption also declined. There was no change in BMI from baseline.

Patient characteristics including medication of participants at follow-up are summarised in ESM Table 2. There was an overall increase in the proportion of participants using medication during the follow-up. In the RC group, the proportion taking antihypertensive drugs had increased from $40 \%$ to $76 \%$ at follow-up, while the proportion taking lipid-lowering drugs had increased from $20 \%$ to $81 \%$. In the IT group, the corresponding findings were increases from $38 \%$ to $82 \%$ and $22 \%$ to $85 \%$. At follow-up, antiglycaemic drugs were taken by $52 \%$ in the RC group and $63 \%$ in the IT group.

Compared with the analysed population, patients with one missing value on CAN tests were older (66.2 years) and included more smokers. Patients with missing values on two or more tests ( $n=22$ ) were older (68.4 years), more were smokers, and their mean BMI was higher. The prevalence of abnormal CAN tests at the follow-up examination, mean values from each test, and median values from the time and frequency domain measures are presented in Table 2 for both groups.

In Fig. 1, the effects of intervention expressed as ORs are presented for the entire study sample and for subgroups. Tests for interaction showed no statistically significant interaction between treatment group (RC/IT) and sex, age, baseline $\mathrm{HbA}_{1 \mathrm{c}}$, baseline total cholesterol and systolic blood pressure.

$\beta$ blockers and hypoglycaemia are known to affect HRV. However, exclusion of patients taking $\beta$ blockers or those who reported episodes of hypoglycaemia did not substantially change the results.

\section{Discussion}

We found no overall effect of intensive multifactorial treatment in general practice, delivered through education and
Table 1 Characteristics at baseline of participants in the Danish arm of ADDITION who had two complete CAN tests

Data are mean (SD) unless otherwise indicated

${ }^{\mathrm{a}}$ Median (25th; 75th percentile) IFCC, International Federation of Clinical Chemistry and Laboratory Medicine

\begin{tabular}{|c|c|c|}
\hline Characteristic & Routine care & Intensive treatment \\
\hline Patients, $n$ & 311 & 466 \\
\hline Male, $n(\%)$ & $178(57 \%)$ & $287(62 \%)$ \\
\hline Age (years) & $59.3(6.6)$ & $59.2(6.8)$ \\
\hline $\mathrm{HbA}_{1 \mathrm{c}}^{\mathrm{a}}(\mathrm{DCCT}, \%)$ & $6.4(6.0 ; 6.9)$ & $6.4(6.0 ; 7.1)$ \\
\hline $\mathrm{HbA}_{1 \mathrm{c}}{ }^{\mathrm{a}}(\mathrm{IFCC}, \mathrm{mmol} / \mathrm{mol})$ & $46(42 ; 52)$ & $46(42 ; 54)$ \\
\hline Systolic blood pressure (mmHg) & $147.1(18.3)$ & $147.1(18.3)$ \\
\hline Diastolic blood pressure $(\mathrm{mmHg})$ & $89.0(11.1)$ & $88.0(10.1)$ \\
\hline Weight (kg) (F;M) & F 83.0 (16.8); M 94.4 (18.1) & F 83.5 (16.5); M 94.7 (17.2) \\
\hline Height (cm) (F;M) & F 163.3 (5.5); M $175.9(8.9)$ & F 163.7 (6.0); M $176.1(8.8)$ \\
\hline BMI $\left(\mathrm{kg} / \mathrm{m}^{2}\right)(\mathrm{F} ; \mathrm{M})$ & F 31.1 (5.8); M 30.5 (5.4) & F 31.1 (5.9); M 30.5 (5.1) \\
\hline Total cholesterol (mmol/l) & $5.69(1.10)$ & $5.59(1.06)$ \\
\hline HDL-cholesterol (mmol/l) & $1.38(0.32)$ & $1.35(0.36)$ \\
\hline Triacylglycerol $(\mathrm{mmol} / \mathrm{l})^{\mathrm{a}}$ & $1.60(1.10 ; 2.40)$ & $1.60(1.16 ; 2.20)$ \\
\hline Smoking daily, $n(\%)$ & $92(30 \%)$ & $137(30 \%)$ \\
\hline Alcohol (units per week) ${ }^{\mathrm{a}}$ & $7(2,14)$ & $7(2,16)$ \\
\hline Microalbuminuria, $n(\%)$ & $36(13 \%)$ & $52(13 \%)$ \\
\hline Macroalbuminuria, $n(\%)$ & $2(1 \%)$ & $8(2 \%)$ \\
\hline Antihypertensive drugs, $n(\%)$ & $123(40 \%)$ & $171(38 \%)$ \\
\hline ACE inhibitors or ATII blockers & $59(19 \%)$ & $82(18 \%)$ \\
\hline$\beta$ blockers & $50(16 \%)$ & $82(18 \%)$ \\
\hline Lipid-lowering drugs, $n(\%)$ & $59(19 \%)$ & $103(22 \%)$ \\
\hline
\end{tabular}


Table 2 At the 6-year follow-up examination, comparison of prevalence ( $\%$ of examined population) of abnormal CAN tests and measures of HRV in the RC and IT group in the Danish cohort of the ADDITION Study

\begin{tabular}{|c|c|c|c|c|}
\hline Variable & $n(\mathrm{RC} / \mathrm{IT})$ & $\mathrm{RC}$ & IT & $p$ value \\
\hline CAN 0 & $243 / 356$ & $77.9(73.2,82.5)$ & $77.2(73.4,81.0)$ & 0.69 \\
\hline CAN early & $47 / 72$ & $15.1(11.1,19.1)$ & $15.5(12.2,18.8)$ & \\
\hline CAN manifest & $22 / 34$ & $7.1(4.2,9.9)$ & $7.3(4.9,9.7)$ & \\
\hline Lying to standing $(30 / 15)$ & & $1.14(1.06 ; 1.23)$ & $1.13(1.06 ; 1.22)$ & 0.43 \\
\hline E:I ratio & & $1.15(1.10 ; 1.22)$ & $1.15(1.09 ; 1.23)$ & 0.65 \\
\hline Valsalva manoeuvre & & $1.46(1.27 ; 1.68)$ & $1.45(1.27 ; 1.65)$ & 0.39 \\
\hline \multicolumn{5}{|l|}{ HRV } \\
\hline SDNN (ms) & & $27.7(19.1 ; 39.8)$ & $26.8(19.6 ; 39.3)$ & 0.52 \\
\hline RMSSD (ms) & & $17.6(11.3 ; 30.1)$ & $17.2(10.55 ; 27.0)$ & 0.28 \\
\hline $\mathrm{aLF}\left(\mathrm{ms}^{2}\right)$ & & $58.3(23.8 ; 139.7)$ & $59.1(25.2 ; 123.0)$ & 0.75 \\
\hline $\mathrm{aHF}\left(\mathrm{ms}^{2}\right)$ & & $37.3(14.2 ; 101.0)$ & $31.3(12.5 ; 86.7)$ & 0.25 \\
\hline Heart rate & & $72.4(12.6)$ & $72.3(12.7)$ & 0.87 \\
\hline
\end{tabular}

Prevalences are presented with $95 \%$ CI, and the rest of the values are presented as median (25th; 75 th percentile), except heart rate (mean and standard deviation).

HF, high-frequency band; LF, low-frequency band; RMSSD, root mean square of the sum of the squares of differences between consecutive R-R intervals ; SDNN, standard deviation from normal to normal intervals

support of general practitioners and practice nurses in evidence-based target-driven management and strict treatment targets/algorithms, on the prevalence of CAN compared with routine care delivered according to national recommendations. However, at the 6-year follow-up examination, participants in both treatment groups in the Danish arm of the ADDITION Trial were overall very well treated. Our study found that, 6 years after a screening-based diagnosis of diabetes, more than one in

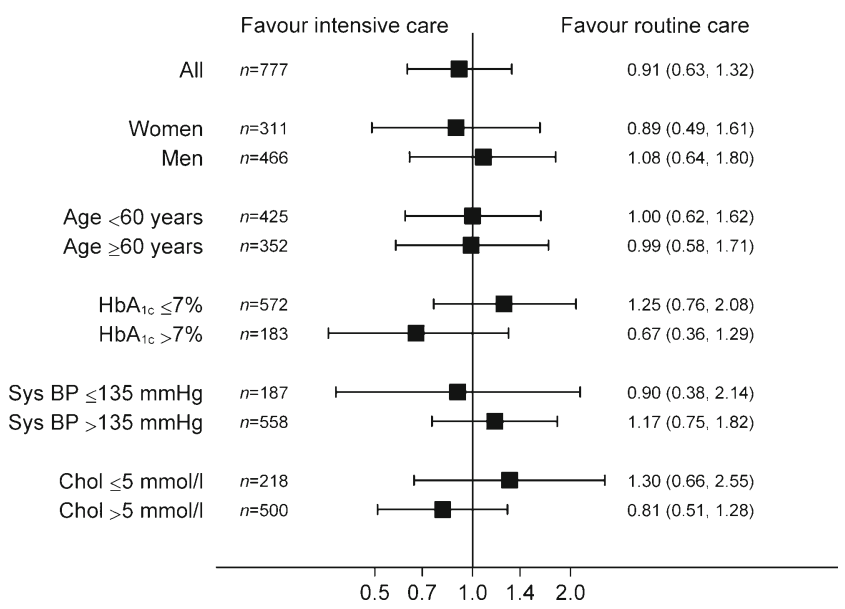

Fig. 1 Effect of intensive multifactorial care in general practice on presence of at least one abnormal CAN test at 6-year follow-up examination compared with routine care expressed as OR $(95 \% \mathrm{CI})$ taking cluster effect into account. The effect is presented for the entire study sample and for subgroups stratified by sex, baseline values for age, $\mathrm{HbA}_{1 \mathrm{c}}$, systolic blood pressure (Sys BP) and total cholesterol (Chol). To convert values for $\mathrm{HbA}_{1 \mathrm{c}}$ in $\%$ into $\mathrm{mmol} / \mathrm{mol}$, subtract 2.15 and multiply by 10.929 seven patients had evidence of early CAN and one in 14 had manifest CAN.

The reported prevalence of CAN in other studies varies considerably according to the diagnostic criteria, assessment modalities and patient selection of each study [12]. In the DiaCAN multicentre study, 524 patients were examined using spectral analyses and predefined CAN tests; $22.1 \%$ had definite CAN (three abnormal tests out of six tests), while $12.2 \%$ had borderline CAN, defined as two abnormal tests [17]. A study including patients from an outpatient diabetes clinic reported a prevalence of $44.3 \%$ among 400 patients with type 2 diabetes when four CAN standard tests were used [18]. Hence, it is not straightforward to compare prevalences with our study. However, it is important to emphasise that our findings show that, even in this group of well-treated patients at an early stage of type 2 diabetes, signs of CAN are present in a sufficiently large proportion to warrant clinical attention. CAN is related to overall mortality. In a meta-analysis, the relative risk of overall mortality associated with CAN was 2.14 (95\% CI 1.83, 2.51) [1], and, in a study on asymptomatic diabetic patients, cardiac autonomic dysfunction had the strongest association with the primary events (i.e. cardiac death and non-fatal myocardial infarction) [19]. Furthermore, based on findings from the ACCORD (Action to Control Cardiovascular Risk in Diabetes) and VADT (Veterans Affairs Diabetes Trial) studies, it has been speculated that diabetic patients with CAN may represent a group where intensive treatment and strict treatment targets could pose a serious risk [20]. In our study, nearly a quarter of the participants had either early or manifest CAN 6 years after screen-detected diabetes diagnosis. 
Therefore testing patients for CAN early in the course of diabetes could potentially supply the clinician with important information on prognosis and treatment. Likewise, in an earlier analysis of the ADDITION-Denmark Study, we found that $34.8 \%$ in the RC group and $30.1 \%$ in the IT group had signs of DPN at the follow-up examination [4]. This confirms that diabetic neuropathy is indeed prevalent among patients in the early stages of type 2 diabetes.

Several risk factors for the development of neuropathy exist [1, 18, 21-23]. However, trial evidence of treatment to prevent the development of CAN in people with type 2 diabetes is sparse $[1,12,24]$. The most salient lesson on prevention of CAN derives from the Steno-2 Study. In the Steno-2 study, intensive multifactorial treatment led to a $69 \%$ reduction in the risk of developing CAN after 7.8 years of follow-up [2], and therefore a multifactorial approach is suggested as the most beneficial [1].

The treatment strategy in the ADDITION Study was a multifactorial, pharmacological and non-pharmacological intervention and was inspired by the strategy used in the Steno-2 Study. However, we found no difference in prevalence of CAN between treatment groups in our study. First, during the follow-up period there was in general and among clinicians a great focus on the treatment of diabetes in Denmark, as the national treatment guidelines were revised in accordance with the results of the Steno-2 Study $[8,9]$. This also led to a substantial improvement in treatment in the RC group. Hence, at follow-up, the two treatment groups were almost equally well treated. We previously speculated that clinicians in the study may use a threshold approach with regard to the prescription of drugs [25]. As the clinicians in the IT group were educated and supported in target-driven patient management, they may have used a lower threshold for prescription than those in the RC group, especially at the beginning of the study when there were no clearly stated national treatment targets for cholesterol and $\mathrm{HbA}_{1 \mathrm{c}}$ to guide RC practitioners. We performed subgroup analyses to explore this threshold hypothesis by testing whether the effect of intensive treatment was present in specific subgroups of patients. However, we found no subgroup effect of age, sex and baseline values of systolic blood pressure, $\mathrm{HbA}_{1 \mathrm{c}}$ or total cholesterol. Hence, we did not find any patient group that might have benefitted additionally from the intensive treatment.

Second, in the Steno-2 Study, the intensively treated patients were managed by a small team of investigators following specific guidelines, whereas patients were treated in many different general practices in the ADDITION Study. Consequently, adherence to guidelines was lower in the ADDITION Study [3]. Third, patients included in the Steno-2 Study had longstanding diabetes and microalbuminuria at inclusion, and they were followed for a longer period. The longer diabetes duration, the longer duration of the study, and the fact that all patients in the Steno-2 Study already had complications at entry all contribute to the higher prevalence of CAN, while the lower prevalence in our study would make it more difficult to observe a difference between the groups even if there was a true treatment effect.

The overall null result of our study may imply that the predisposition to develop diabetic neuropathy in the early stages of diabetes is driven by pathophysiological mechanisms operating earlier in life. On the other hand, considering the early diabetes diagnosis in our participants, we may simply have been dealing with too small a time window to detect any differences.

There are very limited data on the prevalence and prevention of CAN in patients with screen-detected type 2 diabetes. ADDITION-Europe was the first international study on the effects of early detection and intensive treatment of type 2 diabetes. As CAN was only assessed in a subsample of the Danish arm of the trial, this study is unique. All patients were identified and treated in primary care, and the trained staff, who were blinded to the treatment allocation, used broadly accepted and standardised methods for diagnosis. Our study therefore provides an up-to-date and well-documented estimate of the prevalence of CAN in a population with screen-detected diabetes. However, our study is based on a population of white Northern European patients, and one cannot readily generalise these findings to a broader diabetes population. It is possible that different prevalences and treatment effects are applicable in other populations. We did not test for postural fall in blood pressure, which is now recommended as a CAN test. However, an abnormal orthostatic hypotension test result generally occurs late in diabetes and subsequent to abnormalities in heart rate tests [12]. As the study population in the ADDITION Study is a well-treated population with a recent diabetes diagnosis, we think it is acceptable to assume that only a minority of the patients included would have an abnormal orthostatic hypotension test.

We did not control for respiration during supine rest. However, we only used the time and frequency domain measures to compare the two treatment groups.

The patients excluded from the analyses because of missing values were older and more of them were smokers; the reported prevalences may therefore not reflect the true prevalences. The number of patients in the IT group was larger than in the RC group, even though there were no differences in the number and types of practices in the two groups. The practices were randomised before screening and inclusion of patients, but it seems that the intervention allocation enhanced the focus on screening and thereby inclusion of patients in the IT practices. However, the patient characteristics at baseline 
were not substantially different between the two treatment groups; therefore we think that it is reasonable to assume the prevalence of CAN at baseline was equally distributed among the randomisation groups. The reported prevalence differences can therefore be interpreted as differences in cumulative incidence during the follow-up period.

To conclude, signs of CAN were highly prevalent in a well-treated population with screen-detected type 2 diabetes. The prevalence of CAN was not reduced by education and support of general practitioners and practice nurses in evidence-based target-driven management and treatment targets/algorithms compared with routine care according to national guidelines, but at follow-up the participants in the two treatment groups were equally well treated.

Funding ADDITION-Denmark was supported by the National Health Services in the counties of Copenhagen, Aarhus, Ringkøbing, Ribe and South Jutland in Denmark, the Danish Council for Strategic Research, the Danish Research Foundation for General Practice, Novo Nordisk Foundation, the Danish Centre for Evaluation and Health Technology Assessment, the diabetes fund of the National Board of Health, the Danish Medical Research Council, the Aarhus University Research Foundation. The trial has been given unrestricted grants from Novo Nordisk AS, Novo Nordisk Scandinavia AB, Novo Nordisk UK, ASTRA Denmark, Pfizer Denmark, GlaxoSmithKline Pharma Denmark, Servier Denmark A/S and HemoCue Denmark A/S. Parts of the grants from Novo Nordisk Foundation, Danish Council for Strategic Research and Novo Nordisk were transferred to the other centres.

Duality of interest D.R. Witte is employed by Steno Diabetes Center $\mathrm{A} / \mathrm{S}$, which is a research and teaching hospital collaborating with the Danish National Health Service and owned by Novo Nordisk A/S. K. Borch-Johnsen, T. Lauritzen and D.R. Witte hold shares in Novo Nordisk A/S. K. Borch-Johnsen has been an invited speaker with honorarium paid by Eli Lilly, Novo Nordisk, Takeda and SanofiAventis within the past 5 years. T. Lauritzen has given five lectures for the medical industry within the past 2 years.

Contribution statement $\mathrm{MC}$ wrote the manuscript. MC, JF, DRW, NE and AS collected data. MC, JF, DRW and AS researched data. JF, DRW, NE, KBJ, TL and AS reviewed/edited the manuscript. KBJ, TL and AS designed the study and obtained the grants. All authors approved the final version.

\section{References}

1. Vinik AI, Ziegler D (2007) Diabetic cardiovascular autonomic neuropathy. Circulation 115:387-397

2. Gaede P, Vedel P, Larsen N, Jensen GV, Parving HH, Pedersen O (2003) Multifactorial intervention and cardiovascular disease in patients with type 2 diabetes. N Engl J Med 348:383-393

3. Griffin SJ, Borch-Johnsen K, Davies MJ et al (2011) Effect of early intensive multifactorial therapy on 5-year cardiovascular outcomes in individuals with type 2 diabetes detected by screening (ADDITION-Europe): a cluster-randomised trial. Lancet 378:156167
4. Charles M, Ejskjaer N, Witte DR, Borch-Johnsen K, Lauritzen T, Sandbaek A (2011) Prevalence of neuropathy and peripheral arterial disease and the impact of treatment in people with screendetected type 2 diabetes: the ADDITION-Denmark study. Diabetes Care 34:2244-2249

5. Lauritzen T, Griffin S, Borch-Johnsen K, Wareham NJ, Wolffenbuttel BH, Rutten G (2000) The ADDITION study: proposed trial of the cost-effectiveness of an intensive multifactorial intervention on morbidity and mortality among people with type 2 diabetes detected by screening. Int J Obes Relat Metab Disord 24 (Suppl 3):S6-S11

6. Sandbaek A, Griffin SJ, Rutten G et al (2008) Stepwise screening for diabetes identifies people with high but modifiable coronary heart disease risk. The ADDITION study. Diabetologia 51:11271134

7. Tesfaye S, Boulton AJ, Dyck PJ et al (2010) Diabetic neuropathies: update on definitions, diagnostic criteria, estimation of severity, and treatments. Diabetes Care 33:2285-2293

8. Royal College of General Practitioners in Denmark (1999) Type 2 diabetes in general practice: diagnosis and treatment. Royal College of General Practitioners, Copenhagen

9. Royal College of General Practitioners in Denmark (2004) Type 2 diabetes in general practice: an evidence based guideline. Royal College of General Practitioners, Copenhagen

10. Anonymous (1996) Heart rate variability. Standards of measurement, physiological interpretation, and clinical use. Task Force of the European Society of Cardiology and the North American Society of Pacing and Electrophysiology. Eur Heart J 17:354-381

11. Anonymous (1996) Heart rate variability: standards of measurement, physiological interpretation and clinical use. Task Force of the European Society of Cardiology and the North American Society of Pacing and Electrophysiology. Circulation 93:10431065

12. Spallone V, Ziegler D, Freeman R et al (2011) Cardiovascular autonomic neuropathy in diabetes: clinical impact, assessment, diagnosis, and management. Diabetes Metab Res Rev 27:639653

13. Ewing DJ, Clarke BF (1982) Diagnosis and management of diabetic autonomic neuropathy. Br Med J (Clin Res Ed) 285:916-918

14. Spallone V, Bellavere F, Scionti L et al (2011) Recommendations for the use of cardiovascular tests in diagnosing diabetic autonomic neuropathy. Nutr Metab Cardiovasc Dis 21:69-78

15. Ejskjaer N, Fleischer J, Fleischer J, Jacobsen PE, Poulsen PL, Nygaard H (2008) A pocket-size device to detect autonomic neuropathy. J Diabetes Sci Technol 2:1-6

16. Boulton AJ, Vinik AI, Arezzo JC et al (2005) Diabetic neuropathies: a statement by the American Diabetes Association. Diabetes Care 28:956-962

17. Ziegler D, Gries FA, Muhlen H, Rathmann W, Spuler M, Lessmann F (1993) Prevalence and clinical correlates of cardiovascular autonomic and peripheral diabetic neuropathy in patients attending diabetes centers. The Diacan Multicenter Study Group. Diabete Metab 19:143-151

18. Voulgari C, Psallas M, Kokkinos A, Argiana V, Katsilambros $\mathrm{N}$, Tentolouris $\mathrm{N}$ (2011) The association between cardiac autonomic neuropathy with metabolic and other factors in subjects with type 1 and type 2 diabetes. J Diabetes Complications 25:159-167

19. Young LH, Wackers FJ, Chyun DA et al (2009) Cardiac outcomes after screening for asymptomatic coronary artery disease in patients with type 2 diabetes: the DIAD study: a randomized controlled trial. JAMA 301:1547-1555

20. Vinik AI, Maser RE, Ziegler D (2010) Neuropathy: the crystal ball for cardiovascular disease? Diabetes Care 33:16881690 
21. Witte DR, Tesfaye S, Chaturvedi N, Eaton SE, Kempler P, Fuller JH (2005) Risk factors for cardiac autonomic neuropathy in type 1 diabetes mellitus. Diabetologia 48:164-171

22. Dyck PJ, Davies JL, Wilson DM, Service FJ, Melton LJ III, O'Brien PC (1999) Risk factors for severity of diabetic polyneuropathy: intensive longitudinal assessment of the Rochester Diabetic Neuropathy Study cohort. Diabetes Care 22:1479-1486
23. Wiggin TD, Sullivan KA, Pop-Busui R, Amato A, Sima AA, Feldman EL (2009) Elevated triglycerides correlate with progression of diabetic neuropathy. Diabetes 58:1634-1640

24. Pop-Busui R (2010) Cardiac autonomic neuropathy in diabetes: a clinical perspective. Diabetes Care 33:434-441

25. Pauker SG, Kassirer JP (1987) Decision analysis. N Engl J Med $316: 250-258$ 